\title{
Introduction to special issue on cloud and service computing
}

\author{
Jian Yu • Quan Z. Sheng • Yanbo Han
}

Published online: 26 April 2013

(c) Springer-Verlag London 2013

\section{Introduction}

Over the years, the service-oriented paradigm of assembling software systems and applications using Internet-accessible autonomous and platform-independent software components in a loosely coupled manner has been well received by the public for its unprecedented flexibility and interoperability [2-4]. The research area of service delivery and provisioning has gradually matured into the so-called cloud computing by combining the achievements in both utility computing and grid computing. Cloud computing has the potential to reshape the landscape of the IT industry. It brings a brand-new model of IT provisioning and delivery by offering benefits such as zero upfront investment, on-demand services, flexible and elastic capacity, and rapid service deployment [1]. Popular cloud services such as Amazon EC2, Google Compute Engine, and Apple iCloud are now hosted on large-scale data centers and delivered to users through a variety of devices.

However, realizing the full potential of cloud services raises a number of significant challenges, which have not been fully recognized or addressed in the community. One important issue is that large-scale data centers must offer reli-

\section{J. Yu $(\varangle)$}

Faculty of Information and Communication Technologies, Swinburne University of Technology, Melbourne, Australia e-mail: jianyu@swin.edu.au

\section{Q. Z. Sheng}

School of Computer Science, The University of Adelaide, Adelaide, Australia

e-mail: qsheng@cs.adelaide.edu.au

\section{Y. Han}

Research Center for Cloud Computing,

North China University of Technology,

Beijing, People's Republic of China

e-mail: hanyanbo@ncut.edu.cn able and secure services with high QoS standards to satisfy the on-demand needs of users, which spurs the development of novel service security, reliability, and resource virtualization models and algorithms. The other important issue which is largely ignored by the community is end user programing and personalization for cloud services. For example, after cloud services are delivered, how to enable end users to create on-demand situational applications, how to provide personalized services to specific users, just name a few. Providing solutions to these issues may release the full potential of cloud services and bring genuine user expectation and satisfaction.

\section{In this special issue}

This special issue aims at presenting the latest developments, trends, and solutions of cloud services with special focuses on cloud service reliability model, service virtualization, and user-centric services.

The first article by Chen et al., "Resource virtualization methodology for on-demand allocation in cloud computing systems," proposes a resource virtualization model and its on-demand allocation-oriented infrastructure to solve the conflict between resources' tight coupling characteristics and user's multi-granularity requirements. The article by Elfawal-Mansour et al., "Composite web QoS with workflow conditional pathways using bounded sets," proposes a stochastic reliability model of atomic Web services. Some fault tolerance techniques have been proposed using recovery block adaptation to improve the quality of service.

The article by Mobedpour and Ding, "User-centered design of a QoS-based Web service selection system," proposes an expressive and flexible way for non-expert users to define their QoS queries on Web service selection systems. An enhanced selection model that can handle both exact and 
fuzzy requirements and a two-level ranking algorithm are discussed and evaluated. The article by Han et al., "Situational data integration with data services and nested table," proposes a spreadsheet-like programing environment called Mashroom to support situational data integration by nonprofessional users. In Mashroom, various data sources are encapsulated as data services with nested tables as their unified data model both for internal processing and for external uses. Finally, the article by Falcarin et al., "Context data management: an architectural framework for context-aware services," proposes a framework for context data management relying on open standards (XMPP and REST) in order to efficiently collect and deliver context information to mobile devices.

The papers included in this special issue cover several important topics and present some of the key directions in this vibrant and rapidly expanding area of research and development. We hope that the set of selected papers provides the community with a better understanding of the current directions and areas to focus in future and inspires your own work.
Acknowledgments We thank all the authors for considering this special issue as an outlet to publish their research results in the area of cloud and service computing. We also would like to thank the referees who provided very useful and thoughtful feedback to the authors. Finally, we express our gratitude to the Editors-in-Chief, Prof. KweiJay Lin, Prof. Jen-Yao Chung, and Managing Director, Prof. Kuo-Ming Chao, for their kind support, advice, and encouragement throughout the preparation of this special issue.

\section{References}

1. Armbrust Met al (2010) A view of cloud computing. Commun ACM 53(4):50-58

2. Georgakopoulos D, Papazoglou MP (2008) Service-oriented computing. MIT Press, Cambridge

3. Sheng QZ, Benatallah B, Maamar Z, Ngu AHH (2009) Configurable composition and adaptive provisioning of web services. IEEE Trans Serv Comput 2(1):34-49

4. Yu J, Han J, Sheng QZ, Gunarso SO ( 2012) PerCAS: an approach to enabling dynamic and personalized adaptationfor context-aware services. In: Proceedings of the 10th international conference on service-oriented computing (ICSOC 2012), Shanghai, China, p $173-190$ 